\title{
Rotating molecular gas associated with a silhouette disk in the center of the radio galaxy $3 \mathrm{C} 31$
}

\author{
T. Okuda ${ }^{1,2}$, K. Kohno ${ }^{1}$, S. Iguchi ${ }^{3}$ and K. Nakanishi ${ }^{2}$ \\ ${ }^{1}$ Institute of Astronomy, The University of Tokyo, 2-21-1 Osawa, Mitaka, Tokyo, Japan \\ email: okuda@nro.nao.ac.jp \\ ${ }^{2}$ Nobeyama Radio Observatory, Minamimaki, Minamisaku, Nagano, Japan \\ ${ }^{3}$ National Astronomical Observatory of Japan, 2-21-1, Osawa, Mitaka, Tokyo, Japan
}

\begin{abstract}
We have carried out aperture synthesis $\mathrm{CO}(\mathrm{J}=1-0)$ observations of the FRI radio galaxy 3C 31 (NGC 383), using the Nobeyama Millimeter Array (NMA) and the RAINBOW interferometer, which achieves a large collecting area by adding the NRO $45 \mathrm{~m}$ telescope. Our high-resolution $\left(1.9^{\prime \prime} \times 1.4^{\prime \prime}\right)$ CO 3D observations reveal a very massive $\left(\sim 10^{9} M_{\odot}\right)$, circularlyrotating molecular gas ring, which coincides nicely with the silhouette disk seen in the Hubble Space Telescope (HST) optical images. This is the first map depicting the molecular gas distribution and kinematics associated with a silhouette disk in the heart of a radio galaxy.
\end{abstract}

\section{Introduction}

It is now believed that Active galactic nuclei (AGNs) are powered by accretion of interstellar matter (ISM) onto super-massive black holes. Extensive searches for dust features have been made in early type galaxies with and without nuclear activity (Tomita et al. 2000). The extended dust features seen in radio galaxies show various morphologies, i.e., disks, rings, and filamentary or irregular structures with a size scale of a few $100 \mathrm{pc}$ to a few kpc. These results revealed that dust features are prevalent in many $3 \mathrm{C}$ radio galaxies, and suggested that a presence of ISM in the circumnuclear region $(\sim$ a few 100 pc region) of AGNs is indeed inevitable.

HST observations reveal the presence of a silhouette disk with a diameter of $2 \mathrm{kpc}$ in the central region of FR-I radio galaxy 3C 31 (Martel et al. 2000). Detection of abundant molecular gas in $3 \mathrm{C} 31$ has been reported in the course of a $\mathrm{CO}(\mathrm{J}=1-0)$ and $\mathrm{CO}(\mathrm{J}=2-1)$ survey toward $3 \mathrm{C}$ radio galaxies, using the IRAM $30 \mathrm{~m}$ telescope (Lim et al. 2000).

\section{Observations and Results}

The NMA and RAINBOW interferometer observations of $\mathrm{CO}(\mathrm{J}=1-0)$ emission toward the central region of $3 \mathrm{C} 31$. The NMA consists of six $10 \mathrm{~m}$ antennas, and the RAINBOW interferometer combine the Nobeyama $45 \mathrm{~m}$ Radio Telescope with NMA to achieve a very large collecting area.

Fig. 1 displays the optical appearance of the silhouette disk taken with the HST (Fig. 1a), the distribution of CO intensities (Fig. 1b, c), and the mean velocity map along the line of site (Fig. 1d). We find that the distribution of $\mathrm{CO}$ gas in 3C 31 is a ring feature and nicely coincides with the silhouette disk. The molecular gas mass of the disk in $3 \mathrm{C} 31$ is $8.8 \times 10^{8} M_{\odot}$ within a radius of $r<1 \mathrm{kpc}$. The peak gas surface density, $\Sigma_{\text {gas }}$, is $4.0 \times 10^{2} M_{\odot} \mathrm{pc}^{-2}$ at $440 \mathrm{pc}$ from the center. We also find that circular motion dominates the kinematics of the disk. 


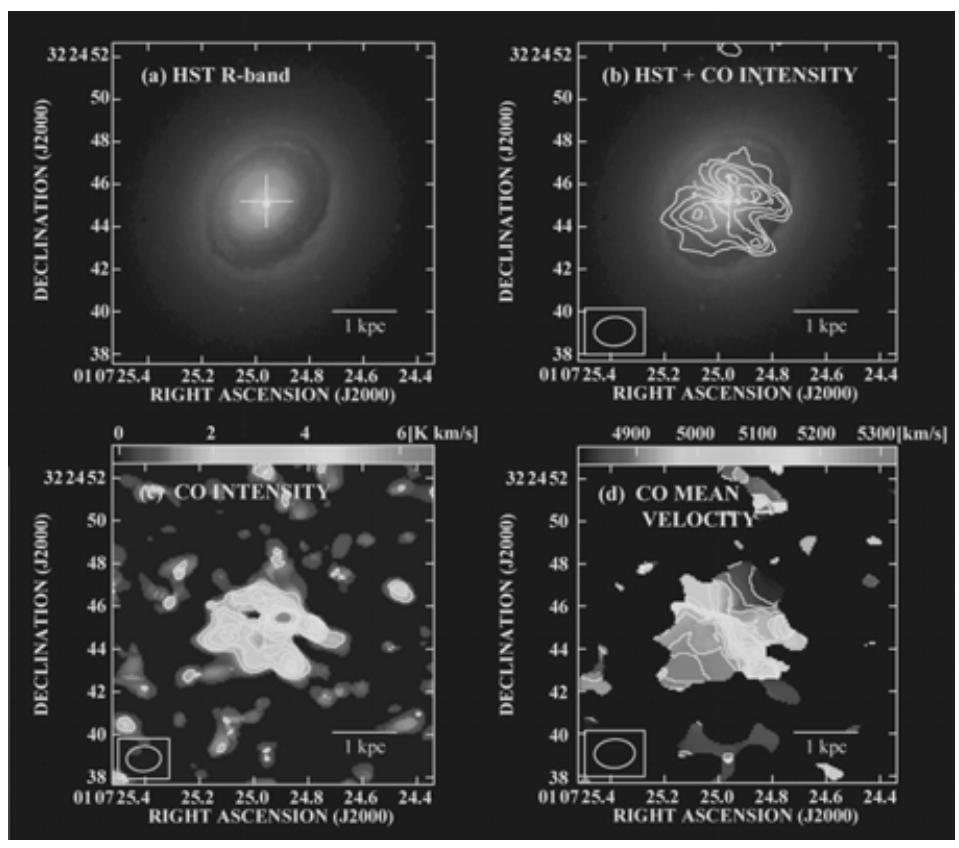

Figure 1. The NMA and RAINBOW maps of $\mathrm{CO}(\mathrm{J}=1-0)$ emission in the radio galaxy $3 \mathrm{C}$ 31. The central cross in figures $(a),(b),(c)$, and $(d)$ marks the peak of $8.4 \mathrm{GHz}$ continuum (Wilkinson et al. 1998). (a) HST R-band image of the center of 3C31. The HST image was obtained from the data archive of the Multimission Archive at STScI. (b) Integrated intensity map (contour) of CO, superposed on a HST image (color). (c) Integrated intensity map of CO. (d) Intensity-weighted mean velocity map of CO.

\section{Discussion}

The total molecular gas mass of the disk in $3 \mathrm{C} 31$ is $2.1 \times 10^{9} M_{\odot}$ within the F.O.V., using the Galactic conversion factor $1.8 \times 10^{20} \mathrm{~cm}^{-2}\left(\mathrm{~K} \mathrm{~km} \mathrm{~s}^{-1}\right)^{-1}$. Since the dust mass derived from IRAS data is $4.0 \times 10^{6} M_{\odot}$ (de Koff et al. 2000), the gas-dust ratio is about 500, which is the same as early-type galaxies (Wiklind et al. 1995), that is, the conversion factor in 3C 31 is comparable to that of the quiescent disk GMCs in the Milky Way.

Spectroscopic studies of 3C 31 show that the optical line ratios within the silhouette disk are not characteristic of photoionization by massive stars (Owen et al. 1990). In this disk, Toomre's Q parameter is much larger than unity. The molecular disk in 3C 31 is gravitationally stable, and we suggest that this is the reason why star formation is inactive there. We propose that a clue to this issue is a gravitational instability of circumnuclear molecular gas disks in AGNs, and suggest that nuclear gas disks in early type hosts tend to be stabilized gravitationally (Kohno et al. 2002).

\section{References}

de Koff, S., et al. 2000, ApJS, 129, 33

Kohno, K., et al. 2002, PASJ, 54, 541

Lim, J., Leon, S., Combes, F., \& Dinh-V-Trung 2000, ApJ, 545, L93

Martel, A. R., Turner, N. J., Sparks, W. B., \& Baum, S. A. 2000, ApJS, 130, 267

Owen, F. N., O'Dea, C. P., \& Keel, W. C. 1990, ApJ, 352, 44

Tomita, A., Aoki, K., Watanabe, M., Tanaka, T., \& Ichikawa, R. 2000, AJ, 120, 123

Wiklind, T., Combes, F., \& Henkel, C. 1995, A\&A, 297, 643

Wilkinson, P. N., et al. 1998, MNRAS, 300, 790 